\title{
Prise en compte des comportements viscoélastiques dans la simulation dynamique des systèmes de freinage
}

\author{
Sylvain Thouvioto $^{1, a}$, Gä̈l Chevallier ${ }^{1}$, Franck Renaud $^{1}$, Jean-Luc Dion ${ }^{1}$ \\ ET RÉmi LEMAIRE ${ }^{2}$ \\ 1 LISMMA - Institut Supérieur de Mécanique de Paris, 3 rue Fernand Hainaut, 93400 Saint-Ouen, France \\ 2 BOSCH Systèmes de freinage, Dépt. NVH, 93700 Drancy, France
}

Reçu le 23 juin 2009, accepté le 23 juin 2009

Résumé - Dans le cadre de la simulation des systèmes de freinage, la mise en évidence de phénomènes tels que le crissement fait appel à des méthodes spécifiques. On s'intéressera ici à l'analyse de stabilité par le calcul des pulsations et modes propres. L'utilisation de dispositifs amortissants « shims » pour la réduction du bruit de crissement implique que les simulations prennent en compte les effets amortissants des matériaux. On utilise pour cela des modèles de matériaux viscoélastiques et en particulier le modèle de Maxwell généralisé. Les travaux ont consisté à choisir et valider des outils (modèles viscoélastiques, formulations éléments finis, ...) et à mettre en place une méthode de simulation. Ils se sont divisés en plusieurs étapes. Dans un premier temps, il a fallu sélectionner, parmi plusieurs modèles mathématiques permettant de décrire la viscoélasticité, le plus adapté à la modélisation de l'amortissement dans les systèmes de freinage. On a étudié les modèles à module complexe, de Kelvin-Voigt et de Maxwell généralisé. Dans un second temps, il a fallu trouver une formulation du problème éléments finis adaptée à l'analyse modale. On a alors utilisé une formulation en modèle d'état. Une hypothèse importante concernant l'égalité des pôles du modèle de Maxwell a permis de limiter la taille du modèle tout en simplifiant sa formulation. Enfin, les méthodes mises en place ont été appliquées à une plaquette de frein dans un premier temps et à un frein complet ensuite. Des analyses paramétriques sur le nombre de modes de projection et l'ordre des modèles de Maxwell ont été menées sur ces deux cas test. Dans le cas de la plaquette de frein, on a effectué une analyse modale complexe en prenant en compte la viscoélasticité du matériau de friction. L'objectif était d'obtenir un recalage de la plaquette, en terme de fréquences et d'amortissements, par rapport à une mesure FRF. Dans le cas du modèle de frein complet, on a effectué une analyse modale complexe en prenant en compte la viscoélasticité des matériaux de friction et du «shim ». Le modèle de frein a également servi de support à la mise en évidence du lien entre la répartition de l'énergie de déformation dans le frein et l'amortissement observé par analyse modale complexe. Pour conclure, on a développé une méthode de simulation utilisant Abaqus ${ }^{\circledR}$, Matlab ${ }^{\circledR}$ pour la phase d'analyse modale complexe avec effets viscoélastiques et Python pour l'interfaçage entre Abaqus ${ }^{\circledR}$ et Matlab ${ }^{\circledR}$. Cette méthode permet de traiter des problèmes d'analyse de stabilité avec la prise en compte de plusieurs matériaux viscoélastiques, sur des modèles de grande dimension, sans surcoût en terme de temps de calcul.

Mots clés : Viscoélasticité / analyse modale complexe / Maxwell généralisé / amortissement / frein / énergie de déformation

\begin{abstract}
Dynamic computation of braking systems including viscoelastic behaviours. In the context of brake system simulation, the highlight of phenomenon like squeal needs specific methods. We will focus on stability analysis by complex modal analysis. The use of damping system called shims for squeal noise reduction implies that simulations take into account the damping of materials. To do this, we use parametric viscoelastic models and particularly the generalised Maxwell model. Work consisted in tools choice and validation (viscoelastic models, finite elements formulations, ...) and in simulation method implementation. It was divided in several steps. In a first step, we have selected, among several mathematical models able to describe viscoelasticity, the best for damping modelisation in brake systems. We studied the complex modulus model, the Kelvin-Voigt model and the generalised
\end{abstract}

${ }^{a}$ Auteur pour correspondance : sylvain.thouviot@supmeca.fr 


\begin{abstract}
Maxwell model. In a second step, we sought a formulation of the finite element problem adapted to the modal analysis. We then used a state space model formulation. A capital hypothesis on the equality of the pole of the generalised Maxwell model permits to reduce the size of the model and in the same time to simplify its formulation. Finally, the developed methods have been applied to simulate a pad and a full brake system. The influence of the number of modes used in model reduction and the order of the Maxwell model has been quantified in parametric studies. In the pad case, we performed a complex modal analysis with friction material considered as viscoelastic. The objective was to update the pad frequencies and damping according to FRF measures. In the brake case, we performed a complex modal analysis with the two friction materials and one shim considered as viscoelastic. This model has also been used to exhibit the link between the strain energy distribution in the brake and the damping level obtained by complex modal analysis. To conclude, we developed a simulation method using Abaqus ${ }^{\circledR}$ and Matlab ${ }^{\circledR}$ for the complex modal analysis with viscoelasticity effects and Python to make data exchange between Abaqus ${ }^{\circledR}$ and Matlab ${ }^{\circledR}$ possible. This method permits to perform stability analysis with several viscoelastic materials, on large models, without degradation of computation time.
\end{abstract}

Key words: Viscoelasticity / complex modal analysis / generalized Maxwell / damping / brake / strain energy

\section{Introduction}

Le contrôle et la prédiction des vibrations et des niveaux de bruit des systèmes mécaniques sont devenus des critères importants dans la conception de nouveaux produits. Dans le cas d'un système de freinage, la source principale d'excitation est le frottement des plaquettes sur le disque à l'origine du bruit de crissement. Les clients étant sensibles à ces bruits indésirables, l'étude du crissement est devenue un axe de recherche prioritaire pour les industriels. Les nombreux travaux publiés sur le sujet montrent que la cause du crissement est souvent un couplage de modes propres dû aux forces de frottement $[1,2]$. La prédiction du comportement vibratoire du frein est réalisée par une méthode classique d'analyse modale complexe sur un modèle discrétisé par éléments finis. Certains matériaux comme les garnitures ou les «shims » utilisés pour l'atténuation des vibrations présentent un comportement viscoélastique. L'impact de l'amortissement sur la stabilité a été mis en évidence par des modèles d'amortissements modaux notamment [3]. Toutefois, l'aspect dépendance à la fréquence caractéristique des comportements viscoélastiques est difficile à prendre en compte dans les codes éléments finis du commerce adaptés aux modèles de tailles industrielles. L'objectif est de développer une méthode d'analyse de stabilité par analyse modale complexe sur des modèles éléments finis de grande taille et viscoélastiques. L'étude est divisée en trois parties : la première traite de la modélisation des comportements viscoélastiques, la seconde traite de la mise en oeuvre de ces modèles dans les analyses modales, la troisième illustre l'application de la méthode sur un cas simple (recalage d'une plaquette) et sur un cas à grand nombre de degrés de liberté (analyse de stabilité sur un frein). Une analyse paramétrique est également menée pour ces deux exemples d'application.

\section{Modélisation des dissipations}

Classiquement, la prise en compte des dissipations dans un modèle éléments finis se traduit par une matrice de raideur complexe et éventuellement dépendante de la fréquence. De manière générale, on s'intéresse au problème aux valeurs propres généralisé suivant :

$$
s_{j}^{2} \underline{\underline{M}} \underline{\phi_{j}}+\underline{\underline{K^{*}}}\left(s_{j}\right) \underline{\phi_{j}}=\underline{0}
$$

Il existe de nombreuses façons de prendre en compte l'amortissement dans un problème de calcul de structures. Dans une approche temporelle, on peut modéliser les fonctions de fluage et de relaxation des matériaux. Dans les approches fréquentielles, le modèle à module complexe, le modèle de Kelvin-Voigt, le modèle de Zener, le modèle de Maxwell généralisé ou encore les modèles à dérivées fractionnaires sont souvent utilisés. Nous nous sommes intéressés à certains de ces modèles dans le but de modéliser le comportement viscoélastique des structures.

La structure pouvant être totalement ou partiellement viscoélastique, on peut toujours distinguer les modules de conservation $\underline{\underline{K_{e}}}$ et les modules de perte $\underline{\underline{K_{\nu}^{*}}}$.

$$
s_{j}^{2} \underline{\underline{M}} \underline{\underline{\phi_{j}}}+\left(\underline{\underline{K_{e}}}+\underline{\underline{K_{\nu}^{*}}}\left(s_{j}\right)\right) \underline{\phi_{j}}=\underline{0}
$$

\subsection{Module complexe}

Le modèle à module complexe a été étudié pour sa simplicité. En effet, il peut s'écrire directement sous la forme d'un problème aux valeurs propres généralisé.

$$
\left[s_{j}^{2} \underline{\underline{M}}+\underline{\underline{K_{e}}}+(1+i \eta) \underline{\underline{K_{\nu}}}\right] \underline{\phi_{j}}=\underline{0}
$$

Dans ce cas, la matrice $\underline{\underline{\underline{K_{\nu}^{*}}}}$ est complexe mais ne dépend pas de la fréquence. Le modèle ne convient donc pas à la modélisation de structures viscoélastiques puisqu'elles présentent un effet de rigidification en fonction de la fréquence.

Notons toutefois que ce modèle d'amortissement structural n'a pas de représentation temporelle et donc pas de réalité physique. 


\section{Nomenclature}

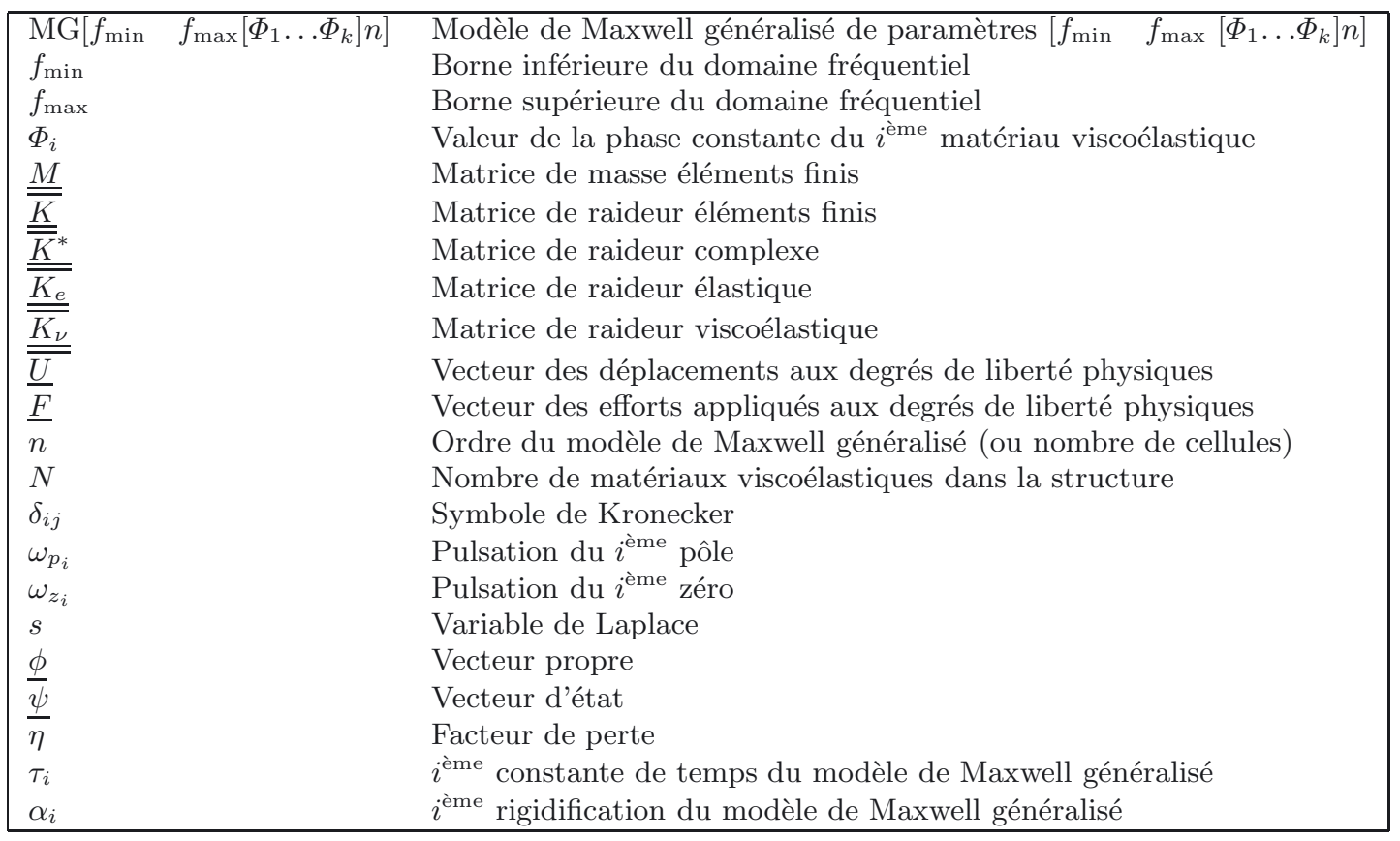

\subsection{Kelvin-Voigt}

Le modèle de Kelvin-Voigt est plus complexe dans le sens où on ne peut pas l'écrire directement sous forme de problème aux valeurs propres généralisé.

$$
\left[s_{j}^{2} \underline{\underline{M}}+\underline{\underline{K_{e}}}+\left(1+\eta s_{j}\right) \underline{\underline{K_{\nu}}}\right] \underline{\phi_{j}}=\underline{0}
$$

Dans ce cas, la raideur est complexe et dépend linéairement de la fréquence. Néanmoins, la dépendance à la fréquence est forte et la raideur non bornée. Cela rend l'utilisation de ce type de modèle valable uniquement sur une bande étroite de fréquence.

\subsection{Maxwell généralisé}

Comme pour Kelvin-Voigt, le modèle de Maxwell généralisé ne s'écrit pas directement sous la forme d'un problème aux valeurs propres généralisé. On peut noter que le modèle de Maxwell généralisé est très similaire à d'autres modèles tels que ADF [4] ou ATF [5]. La formulation (5) utilisée ici a été proposée précédemment dans l'article [6].

$$
\left[s_{j}^{2} \underline{\underline{M}}+\underline{\underline{K_{e}}}+\left(1+\sum_{i=1}^{n} \frac{\alpha_{i} \tau_{i} s_{j}}{1+\tau_{i} s_{j}}\right) \underline{\underline{K_{\nu}}}\right] \underline{\phi_{j}}=\underline{0}
$$

Cette fois, la matrice $\underline{K}_{\nu}^{*}$ est complexe et dépend de la fréquence. Le modèle $\overline{\overline{\text { fait }}}$ intervenir de nombreux paramètres $\alpha_{i}$ et $\tau_{i}, i \in\{1, n\}$. Il permet de prendre en compte relativement facilement la rigidification ainsi que l'amortissement.
Parmi les modèles étudiés, seul le modèle de Maxwell généralisé est capable de représenter convenablement la viscoélasticité sur un domaine de fréquence « large » comme l'impose l'application au crissement de frein.

\section{Remarque :}

On ne considère pas les modèles à dérivées fractionnaires dans ces travaux. Malgré le nombre plus faible de paramètres par rapport à un modèle de Maxwell généralisé, ces modèles sont mal adaptés à une formulation en modèle d'état. L'alternative d'un développement en série conduit à un modèle de Maxwell généralisé.

\subsubsection{Calcul des paramètres de Maxwell}

Le modèle de Maxwell généralisé nécessite le calcul préalable de paramètres qui détermine la forme du modèle (phase et rigidification associée).

La méthode utilisée est basée sur l'identification d'une phase expérimentale donnée. On identifie les paramètres du modèle permettant de représenter au mieux la phase.

Pour identifier cette phase, on utilise la formule (6) extraite du livre d'Oustaloup [7] qui donne la phase d'un modèle de Maxwell formulé avec des pôles et des zéros (cf. formule 5,44 du livre [7]).

$$
\Phi=\sum_{i=1}^{n}\left(\arctan \left(\frac{\omega}{\omega_{z_{i}}}\right)-\arctan \left(\frac{\omega}{\omega_{p_{i}}}\right)\right)
$$

Le calcul de la position optimale des pôles et des zéros est abordé dans les travaux de Franck Renaud [10] mais reste un problème ouvert. Une fois les pôles et zéros calculés, on 
détermine les paramètres du modèle de Maxwell par les formules (7) et (8). Ce passage entre l'écriture en pôles et zéros et l'écriture en constantes de temps et rigidifications est exposé dans la thèse de Jean-Luc Dion [8].

$$
\begin{gathered}
\tau_{i}=\frac{1}{\omega_{p_{i}}} \\
\alpha_{i}=\prod_{j=1}^{n} \frac{\omega_{p_{j}}\left(\omega_{p_{i}}-\omega_{z_{j}}\right)}{\omega_{z_{j}}\left(\omega_{p_{i}}+\omega_{p_{j}}\left(\delta_{i j}-1\right)\right)}
\end{gathered}
$$

\section{Analyse modale complexe}

Dans cette partie, on étudie les propriétés amortissantes des matériaux en exploitant les parties réelles et imaginaires des valeurs propres obtenues par analyse modale complexe.

\subsection{Formulation en modèle d'état}

Nous avons vu que l'équation dynamique d'une structure viscoélastique modélisée par le modèle de Maxwell généralisé peut se mettre sous la forme (5). Il ne s'agit pas d'un problème aux valeurs propres généralisé. Cette formulation n'est donc pas exploitable directement en analyse modale. Pour cela, on considère une formulation en modèle d'état. L'équation (9) donne un exemple d'écriture en modèle d'état pour un modèle de Maxwell généralisé d'ordre $n=1$.

$$
\begin{aligned}
& s_{j}\left[\begin{array}{ccc}
-\tau_{1} M & 0 & 0 \\
0 & I & 0 \\
0 & 0 & I
\end{array}\right] \psi_{j}= \\
& {\left[\begin{array}{ccc}
M & \tau_{1}\left(K_{e}+\left(1+\alpha_{1}\right) K_{\nu}\right) & K_{e}+K_{\nu} \\
I & 0 & 0 \\
0 & I & 0
\end{array}\right] \psi_{j}}
\end{aligned}
$$

le vecteur d'état $\psi_{j}$ étant défini par :

$$
\psi_{j}=\left[\begin{array}{c}
s_{j}^{2} \phi_{j} \\
s_{j} \phi_{j} \\
\phi_{j}
\end{array}\right]
$$

On est ramené à un problème aux valeurs propres généralisé de taille supérieure (multipliée par $n+2$ par rapport au problème de départ). On voit alors que la taille du modèle devient critique pour des ordres de Maxwell généralisé élevés. Il est alors indispensable de travailler sur des modèles réduits. Dans le cadre de cette étude, on propose une projection du modèle dans une base de Ritz constituée des modes propres réels de la structure considérée comme totalement élastique. Cette solution donne de bons résultats [9] pour les structures faiblement amorties.

\subsection{Prise en compte de plusieurs matériaux}

Les formulations présentées jusqu'ici ne prennent en compte qu'un seul matériau viscoélastique, la structure pouvant être partiellement ou totalement viscoélastique. Or l'application de la méthode proposée à un cas industriel tel que le système de freinage impose la possibilité de considérer plusieurs matériaux viscoélastiques (matériaux de friction, « shims»).

On considère une structure composée de $N$ matériaux viscoélastiques modélisés par un modèle de Maxwell généralisé. En reprenant le formalisme de l'équation (5), on peut écrire l'équation dynamique sous la forme :

$$
s_{j}^{2} M \phi_{j}+K_{e} \phi_{j}+\sum_{k=1}^{N}\left[\left(1+\sum_{i=1}^{n} \frac{\alpha_{i}^{k} \tau_{i}^{k} s_{j}}{1+\tau_{i}^{k} s_{j}}\right) K_{\nu}^{k}\right] \phi_{j}=0
$$

On peut remarquer que dans le cas d'un système à 1 degré de liberté, cela reviendrait à considérer $N$ matériaux travaillant en parallèles. La raideur équivalente est alors obtenue par sommation des raideurs propres à chaque matériau. Une vision en terme de somme d'énergies de déformation est également équivalente.

Si on écrit le problème sous forme de modèle d'état, il sera de taille $N \times n+2$ fois la taille du modèle éléments finis de départ. Dans ce cas, même en travaillant avec un modèle réduit, les modèles sont trop grands et présentent des problèmes de conditionnement.

\subsection{Simplification par pôles communs}

Afin de résoudre le problème soulevé à la question précédente, on formule l'hypothèse de travail suivante : «Les constantes de temps $\tau_{i}$ sont égales pour tous les matériaux viscoélastiques » Par conséquent, l'équation (11) peut s'écrire :

$$
s_{j}^{2} M \phi_{j}+K_{e} \phi_{j}+\sum_{k=1}^{N}\left[\left(1+\sum_{i=1}^{n} \frac{\alpha_{i}^{k} \tau_{i} s_{j}}{1+\tau_{i} s_{j}}\right) K_{\nu}^{k}\right] \phi_{j}=0
$$

Le modèle d'état associé a la même taille qu'un modèle à 1 matériau viscoélastique.

Les travaux de Renaud [10] ont montré que cette hypothèse n'est pas une contrainte forte sur la détermination de la position des pôles et des zéros pour estimer un ensemble de courbes de phase expérimentales (cf. Fig. 1).

\section{Applications}

On dispose maintenant d'une formulation éléments finis par modèle d'état d'un problème de dynamique des structures faisant intervenir des matériaux viscoélastiques. Le traitement de problèmes d'analyse modale complexe formulés en modèle d'état ne peut pas se faire directement dans Abaqus ${ }^{\circledR}$, qui est le code de calcul utilisé par Bosch ${ }^{\circledR}$ Système de freinage, notamment 


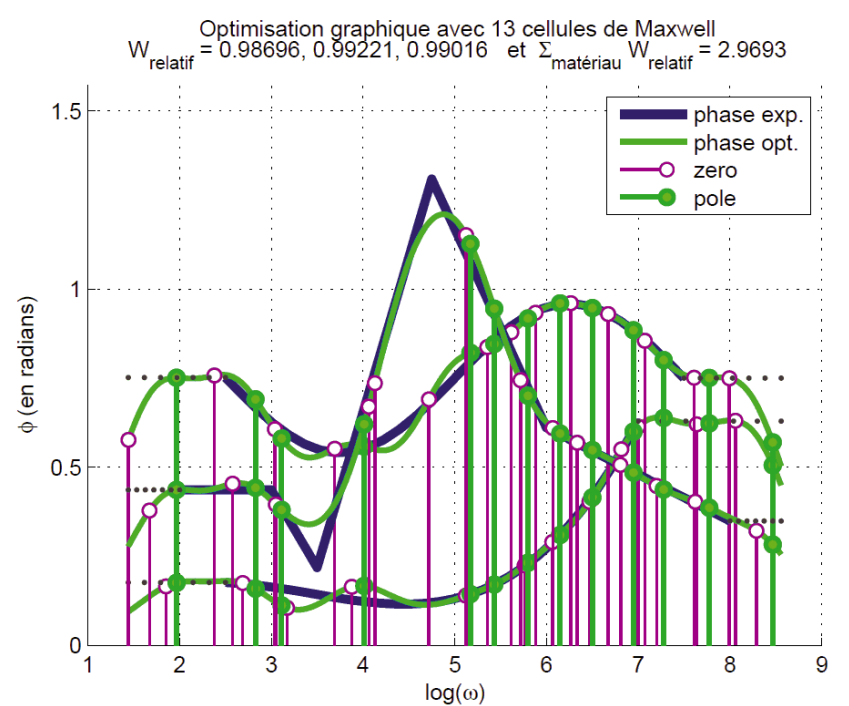

Fig. 1. Estimation de phases quelconques différentes pour trois parties viscoélastiques avec pôles égaux.

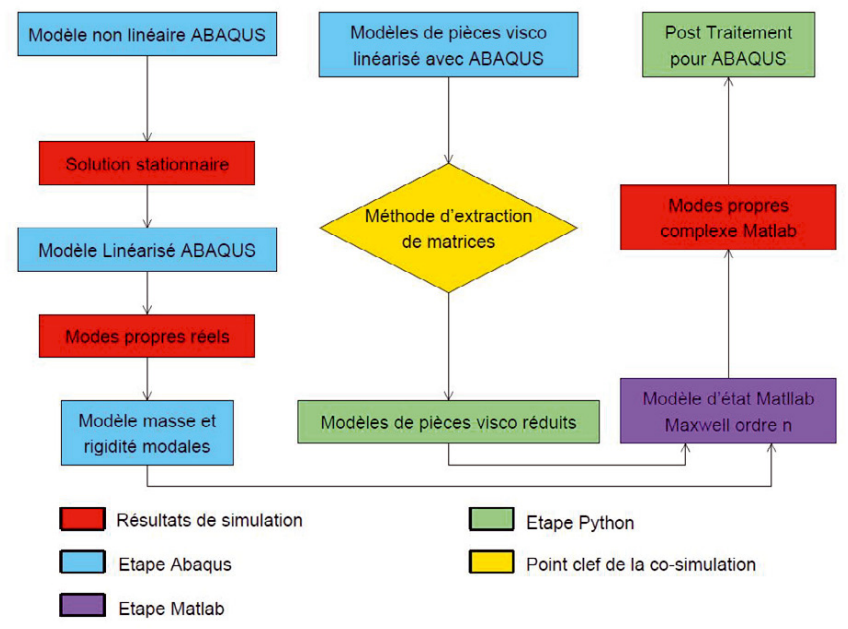

Fig. 2. Principe de la simulation mixte Abaqus ${ }^{\circledR} /$ Matlab $^{\circledR}$.

car les matériaux utilisés ne sont pas isotropes [11]. Il est alors nécessaire de considérer une simulation mixte Abaqus ${ }^{\circledR} /$ Matlab $^{\circledR}$. L'idée est d'effectuer la majeure partie de la simulation dans Abaqus ${ }^{\circledR}$ (mise en pression du frein, mise en rotation du disque, analyse modale réelle). L'ensemble des données nécessaires au calcul des modes complexes est ensuite transféré vers Matlab ${ }^{\circledR}$ par un programme Python. La figure 2 en illustre ce principe.

\subsection{Plaquette}

Dans cette partie, on présente quelques résultats obtenus sur un modèle de plaquette. Il s'agit d'une plaquette extérieur T7 comportant un seul matériau viscoélastique (la garniture). La figure 3 montre le modèle éléments finis utilisés.

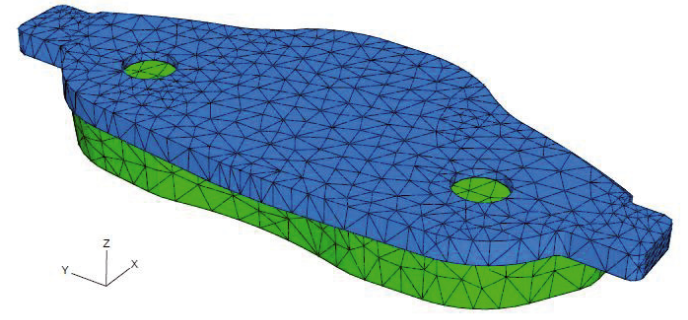

Fig. 3. Modèle de plaquette de frein T7.

Tableau 1. Recalage en fréquence d'une plaquette T7.

\begin{tabular}{cccc}
\hline \multicolumn{4}{c}{ Recalage en fréquence } \\
\hline & $f_{\text {FRF }}(\mathrm{Hz})$ & $f_{\text {calcul }}(\mathrm{Hz})$ & Ecart \\
\hline Mode 1 & 2461 & 2485 & $0,99 \%$ \\
Mode 2 & 4264 & 4239 & $-0,58 \%$ \\
Mode 3 & 4979 & 4988 & $0,17 \%$ \\
Mode 4 & 7512 & 7492 & $-0,27 \%$ \\
Mode 5 & 7796 & 7844 & $0,62 \%$ \\
\hline
\end{tabular}

Tableau 2. Recalage en amortissement d'une plaquette T7.

\begin{tabular}{cccc}
\hline \multicolumn{4}{c}{ Recalage en amortissement } \\
\hline & $\xi_{\text {FRF }}(\%)$ & $\xi_{\text {calcul }}(\%)$ & Ecart \\
\hline Mode 1 & 1,21 & 1,69 & $39,67 \%$ \\
Mode 2 & 1,49 & 1,50 & $0,67 \%$ \\
Mode 3 & 1,78 & 1,40 & $-21,35 \%$ \\
Mode 4 & 1,39 & 1,50 & $7,91 \%$ \\
Mode 5 & 1,67 & 1,50 & $-10,18 \%$ \\
\hline
\end{tabular}

L'objectif était de procéder à un recalage des paramètres matériaux de la plaquette, en fréquence et amortissement, par rapport à des résultats de mesure FRF.

On présente dans les tableaux 1 et 2 les résultats d'un recalage en fréquence et amortissement pour les 5 premiers modes propres de la plaquette. Le calcul Abaqus ${ }^{\circledR}$ est conduit avec les données statiques du matériau. On applique le modèle viscoélastique MG[0.01 20000 [6] 7].

On voit que le modèle proposé permet un très bon recalage en fréquence (moins de $1 \%$ d'écart sur les 5 premiers modes) tout en ayant un recalage très correct sur les amortissements. En effet, en tenant compte de la difficulté de mesure de l'amortissement ainsi que de la difficulté de modélisation, un écart de moins de $40 \%$ sur les 5 premiers modes est tout à fait acceptable.

\subsection{Frein}

Dans cette partie, on présente quelques résultats obtenus sur un modèle de frein complet. Il s'agit d'analyses modales complexes avec prise en compte des matériaux viscoélastiques des deux garnitures et du «shim ». Dans un premier temps, les calculs sont faits sans prise en compte du contact plaquettes-disque puis avec le contact.

Il s'agit d'un modèle non recalé destiné à l'illustration de la prise en compte de la viscoélasticité sur un modèle industriel. 


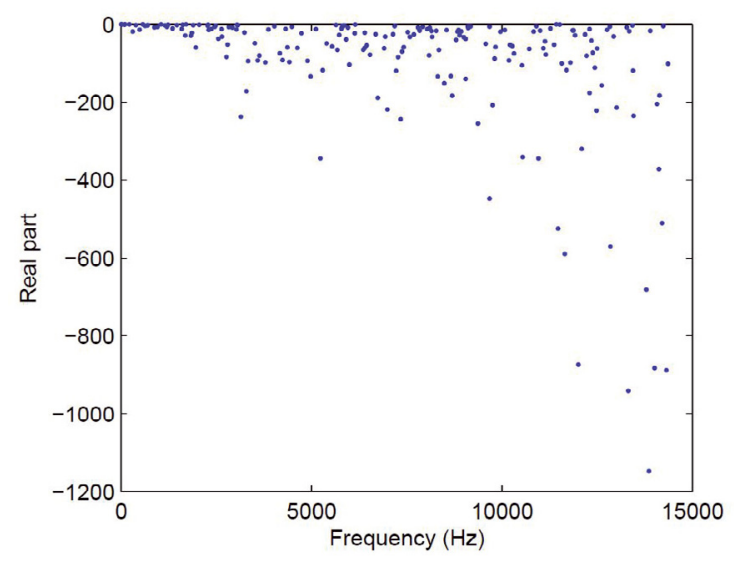

Fig. 4. Partie réelle pour chaque mode propre.

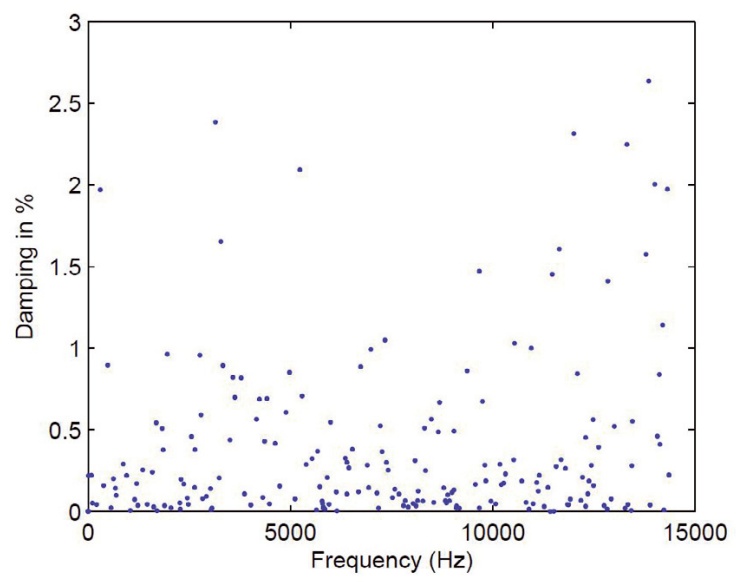

Fig. 5. Amortissement pour chaque mode propre.

\subsubsection{Simulation sans contact}

Dans ce cas, il n'y a aucun couplage tangentiel entre les plaquettes et le disque. Comme il s'agit de la seule source d'instabilité dans le système, on n'observera pas de modes instables.

Les figures 4 et 5 illustrent l'impact de la viscoélasticité sur, respectivement, les parties réelles et les amortissements.

On observe une diminution globale de la partie réelle des valeurs propres complexes. Cela se traduit aussi par l'apport d'amortissement dans la structure. Le résultat obtenu sur cette structure partiellement viscoélastique est tout à fait celui attendu. De plus, l'ordre de grandeur des amortissements obtenus correspond à celui observé dans le cas d'un calcul sur une plaquette seule (cf. Tab. 2).

Il est également intéressant de noter que tous les modes ne sont pas affectés de la même façon par la viscoélasticité. Dans la Section 4.2.3, on établit le lien entre l'amortissement observé et la répartition d'énergie de déformation.

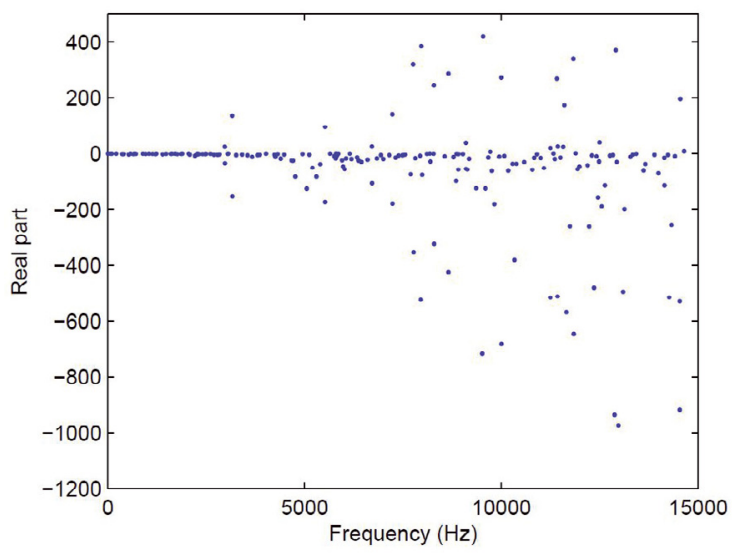

Fig. 6. Partie réelle pour chaque mode propre.

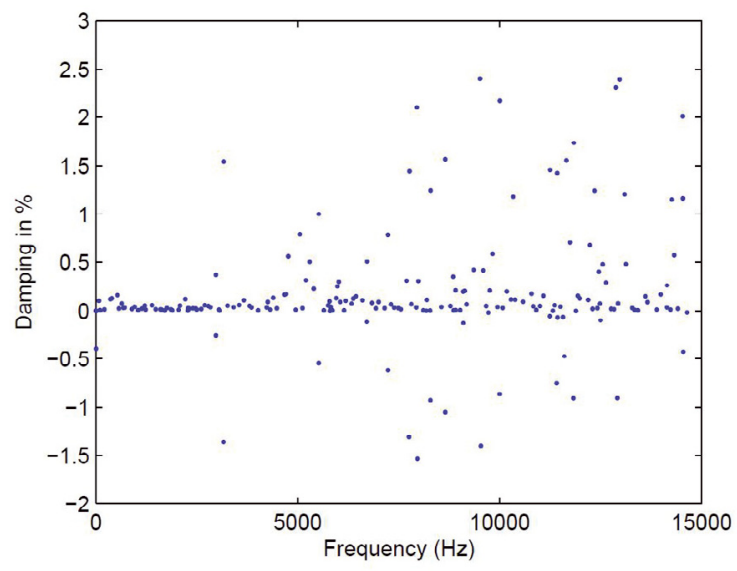

Fig. 7. Amortissement pour chaque mode propre.

\subsubsection{Simulation avec contact}

Dans ce cas, le couplage tangentiel entre les plaquettes et le disque est pris en compte (on n'exposera pas le calcul de la matrice de raideur de couplage). Le modèle de contact tangentiel ne correspond pas à celui de Abaqus ${ }^{\circledR}$. On ne comparera donc pas de résultats d'analyse modale complexe obtenus avec Matlab ${ }^{\circledR}$ et Abaqus ${ }^{\circledR}$.

De la prise en compte du couplage tangentiel résulte l'apparition de modes instables. Cela se traduit par des parties réelles positives (cf. Fig. 6) ou des amortissements négatifs (cf. Fig. 7).

Afin de mieux mettre en évidence l'impact de la viscoélasticité sur les modes instables, on superpose les amortissements négatifs obtenus avec et sans viscoélasticité. Le résultat obtenu est présenté sur la figure 8.

On observe globalement une diminution du niveau de l'instabilité avec la viscoélasticité. En effet, l'amortissement injecté par le modèle de Maxwell généralisé tend à compenser l'amortissement négatif des modes instables. La présence de rigidification dans le modèle de Maxwell se traduit par un décalage fréquentiel des modes amortis. Il est alors difficile de conclure sur l'évolution du niveau d'instabilité d'un mode en particulier. Il faut 


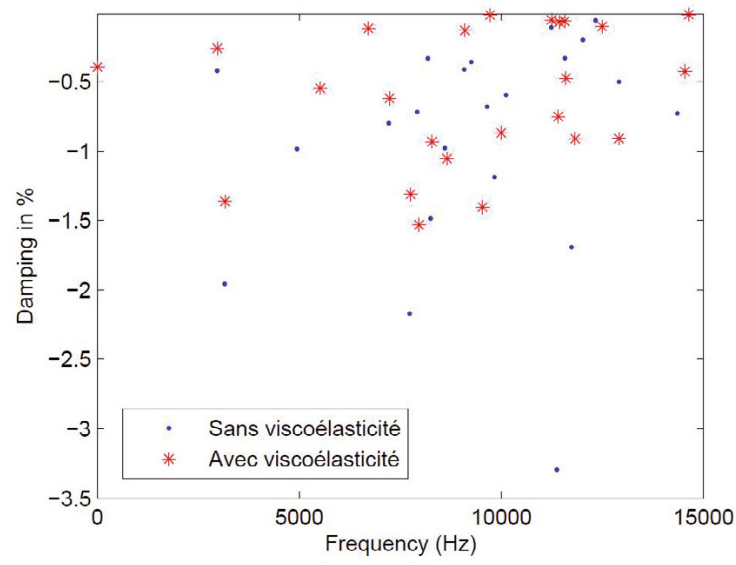

Fig. 8. Comparaison de l'amortissement avec et sans viscoélasticité pour chaque mode propre.

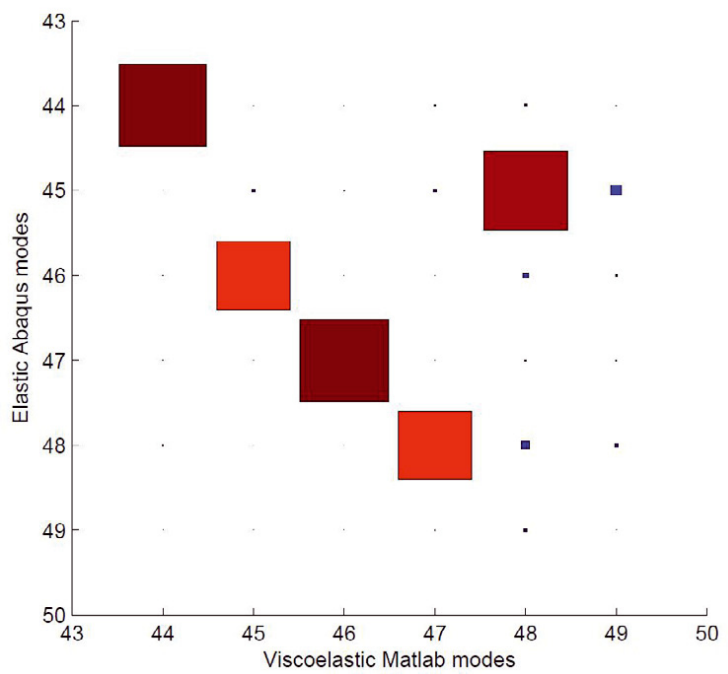

Fig. 9. Corrélation des modes élastiques Abaqus ${ }^{\circledR}$ et des modes « viscoélastiques $»$ Matlab $^{\circledR}$.

également garder à l'esprit que l'ajout d'amortissement dans un système n'est pas toujours source de stabilisation. Ce phénomène est illustré dans [12].

\subsubsection{Lien avec les énergies de déformation}

Pour mettre en évidence le lien entre l'amortissement et la répartition d'énergie dans le frein, on va s'intéresser à 2 modes particuliers. Le premier (mode 46) est faiblement amorti tandis que le second (mode 48) l'est fortement.

Pour connaître le numéro des modes élastiques correspondants (calculés précédemment par Abaqus ${ }^{\circledR}$ ), on trace le diagramme de MAC présenté sur la figure 9 .

On voit qu'il y a un phénomène d'inversion de modes qui vient de l'ajout du modèle viscoélastique. En effet, en plus d'apporter de l'amortissement, il y a un effet de rigidification qui va décaler plus ou moins les modes amortis.

Le mode «viscoélastique » 46 correspond au mode élastique 47.

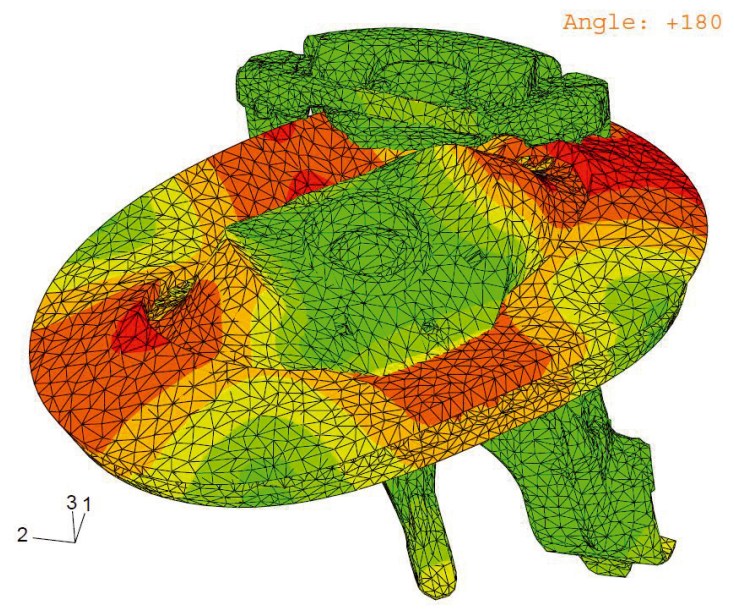

Fig. 10. Déformée du mode 46.

Tableau 3. Récapitulatif de l'énergie de déformation pour le mode 46 d'un frein A5X.

\begin{tabular}{cc}
\hline Pièce & Ratio $E_{d}$ \\
\hline Modèle complet & $100,00 \%$ \\
Chape & $0,33 \%$ \\
Etrier & $0,06 \%$ \\
Disque & $91,54 \%$ \\
Moyeu & $4,91 \%$ \\
Plaquette intérieure & $0,80 \%$ \\
Porte fusée & $1,54 \%$ \\
Plaquette extérieure & $0,79 \%$ \\
Piston & $0,01 \%$ \\
Ensemble viscoélastique & $1,50 \%$ \\
\hline
\end{tabular}

Le mode « viscoélastique » 48 correspond au mode élastique 45.

On commence par étudier le mode « viscoélastique » 46 qui présente un amortissement faible $(3045 \mathrm{~Hz}$ et 0,012\%). Sa déformée est visible sur la figure 10 .

On établit le récapitulatif de l'énergie de déformation $E_{d}$ contenue dans chaque pièce du frein et on calcule le ratio d'énergie contenue dans chaque pièce par rapport à l'énergie totale. Le résultat est présenté dans le tableau 3.

On constate que la majeure partie de l'énergie de déformation est contenue dans le disque (on le voit aussi sur la déformée Fig. 10). La faible quantité d'énergie contenue dans l'ensemble des parties viscoélastiques est en accord avec l'amortissement faible pour ce mode.

On regarde maintenant un mode plus amorti. Il s'agit du mode « viscoélastique » $48(3148 \mathrm{~Hz}$ et $2,385 \%$ d'amortissement). Sa déformée est visible sur la figure 11.

Comme pour le mode précédent, on établit le récapitulatif de l'énergie de déformation $E_{d}$ contenue dans chaque pièce du frein et on calcule le ratio d'énergie contenue dans chaque pièce par rapport à l'énergie totale. Le résultat est présenté dans le tableau 4.

On constate cette fois que la majeure partie de l'énergie de déformation est contenue dans les parties viscoélastiques (et principalement la plaquette intérieure). Ceci est en accord avec l'observation visuelle 


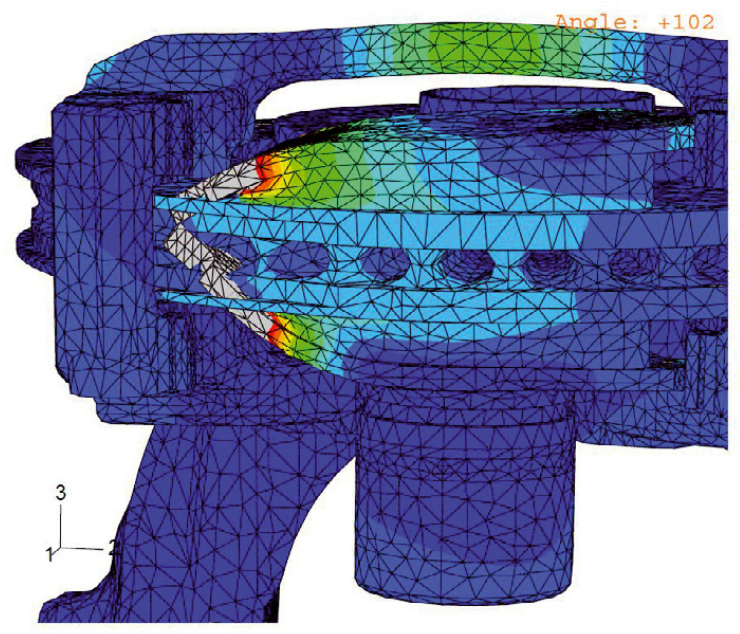

Fig. 11. Déformée du mode 48.

Tableau 4. Récapitulatif de l'énergie de déformation pour le mode 48 d'un frein A5X.

\begin{tabular}{cc}
\hline Pièce & Ratio $E_{d}$ \\
\hline Modèle complet & $100,00 \%$ \\
Chape & $0,94 \%$ \\
Etrier & $3,75 \%$ \\
Disque & $4,07 \%$ \\
Moyeu & $0,06 \%$ \\
Plaquette intérieure & $76,77 \%$ \\
Porte fusée & $0,25 \%$ \\
Plaquette extérieure & $13,81 \%$ \\
Piston & $0,35 \%$ \\
Ensemble viscoélastique & $86,01 \%$ \\
\hline
\end{tabular}

de la déformée (Fig. 11) qui montre un mode de plaquette, et avec l'amortissement important obtenu pour ce mode.

La correspondance directe entre la répartition d'énergie de déformation dans le frein et l'amortissement permet d'envisager une autre façon de traiter l'amortissement. En effet, on pourrait choisir d'affecter une valeur d'amortissement à chaque partie viscoélastique (et même à chaque pièce métallique du frein) pondérée par le ratio d'énergie de déformation contenue dans celle-ci. Il ne s'agirait pas de reproduire les effets de la viscoélasticité car l'effet de rigidification ne serait pas pris en compte, mais bien de modéliser l'amortissement dans le système sans passer par un modèle mathématique complexe.

En revanche, il n'est pas certain que le calcul et le post-traitement des énergies de déformation soient moins coûteux que l'analyse modale complexe avec un modèle de Maxwell.

\section{Analyses paramétriques}

Afin réduire les temps de calcul, on travaille avec des modèles réduits par projection sur une base de Ritz constituée des modes propres réels de la structure élastique. Comme cette base est tronquée, il est nécessaire

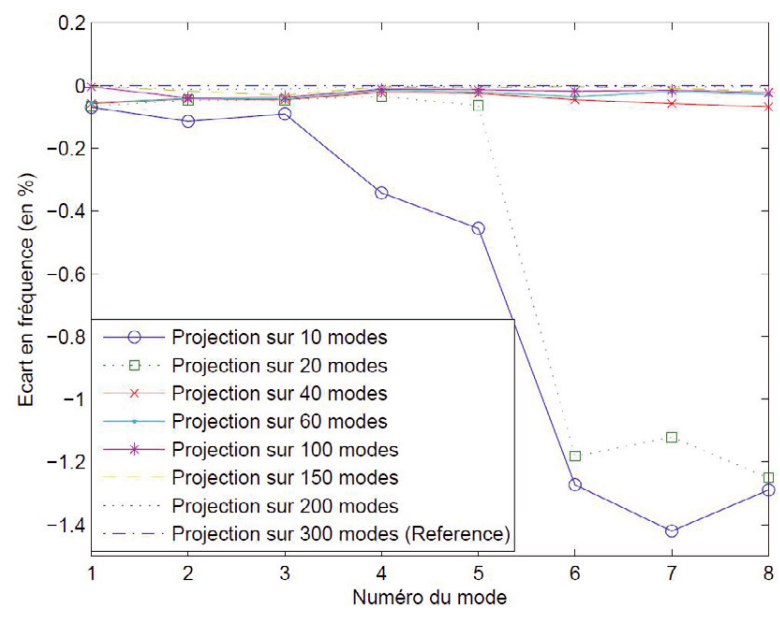

Fig. 12. Ecart en fréquences calculé par analyse modale complexe pour chaque modèle réduit.

d'étudier l'impact de la troncature sur les résultats d'analyse modale complexe.

L'influence du nombre de cellules de Maxwell est également étudiée.

\subsection{Plaquette}

On considère le modèle de plaquette T7 décrit dans la Section 4.1.

\subsubsection{Influence du nombre de modes de projection}

La méthode consiste à extraire successivement 10, 20, $40,60,100,150,200$ puis 300 modes réels et à projeter le modèle sur ces bases. On calcule alors les modes complexes pour chaque modèle réduit. On prend en compte la viscoélasticité par un modèle de Maxwell généralisé d'ordre 4 dont les paramètres sont : MG[5 200003 4]. On obtient alors les fréquences et amortissements de tous les modes pour chaque modèle réduit.

On s'intéresse uniquement aux 8 premiers modes de la plaquette. On trace l'écart en pour cent entre les résultats de chaque modèle réduit et les résultats du dernier (projeté sur 300 modes). Les résultats en fréquence et en amortissement sont illustrés respectivement sur les figures 12 et 13 .

On observe un effet de convergence vers la référence avec l'augmentation du nombre de modes de projection. On peut considérer qu'il faut un minimum de 40 modes pour obtenir un modèle réduit correct (pour étudier les 8 premiers modes).

De plus, on remarque que l'ordre de grandeur des écarts entre 10 et 40 modes est le même que celui du recalage présenté à la Section 4.1. Il est donc nécessaire d'être vigilant quand aux nombres de modes retenus pour la réduction du modèle. 


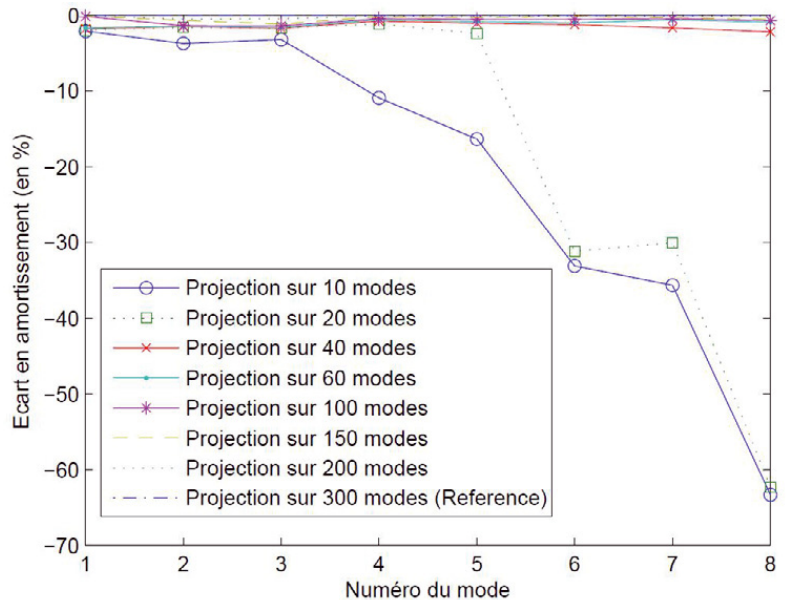

Fig. 13. Ecart en amortissements calculé par analyse modale complexe pour chaque modèle réduit.

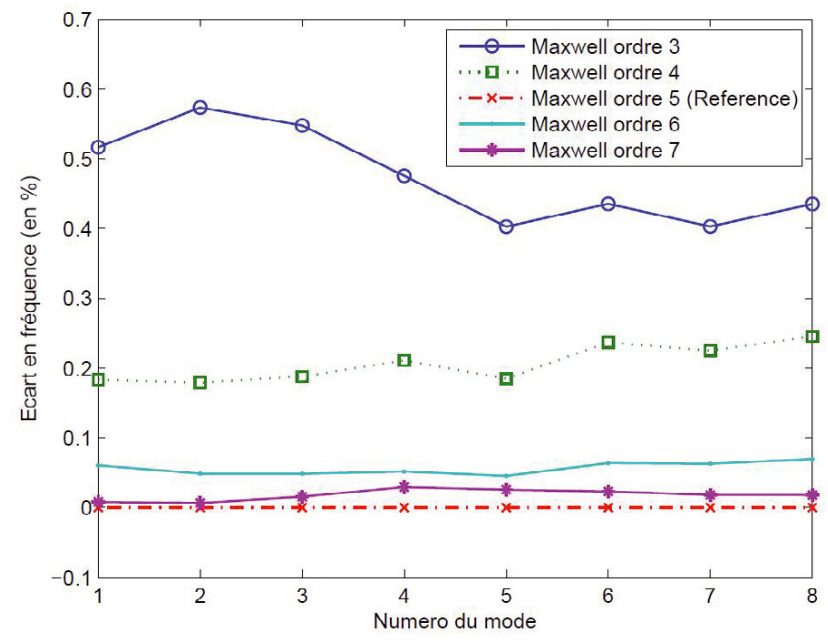

Fig. 14. Effet de l'ordre du modèle de Maxwell sur les fréquences.

\subsubsection{Influence du nombre de cellules de Maxwell}

Afin de s'affranchir des problèmes liés à la réduction du modèle, on travaille avec un modèle projeté sur 60 modes réels. La méthode consiste alors à fixer un intervalle de fréquence de 3 décades (de $20 \mathrm{~Hz}$ à $20000 \mathrm{~Hz}$ ) et une phase constante à 3 degrés. On calcule successivement les fréquences et amortissements par analyse modale complexe en faisant varier l'ordre du modèle de Maxwell généralisé de 3 (1 cellule par décade) à 7 . On s'intéresse à l'influence de ce paramètre sur l'oscillation de la phase du modèle de Maxwell ainsi que son impact sur les fréquences (cf. Fig. 14) et amortissements (cf. Fig. 15).

La référence correspond à l'ordre 5 car pour les ordres supérieurs la phase du modèle de Maxwell n'oscille plus de façon régulière et avec une amplitude constante (cf. Fig. 16). Cela est dû à un mauvais positionnement des pôles et des zéros car la méthode d'optimisation utilisée ne permet pas le contrôle de leur position. Un algorithme

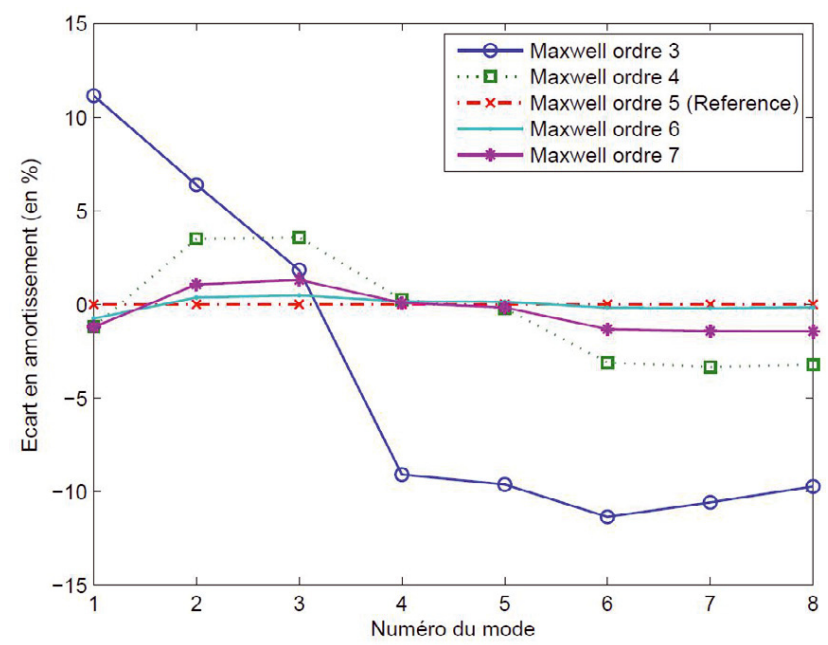

Fig. 15. Effet de l'ordre du modèle de Maxwell sur les amortissements.

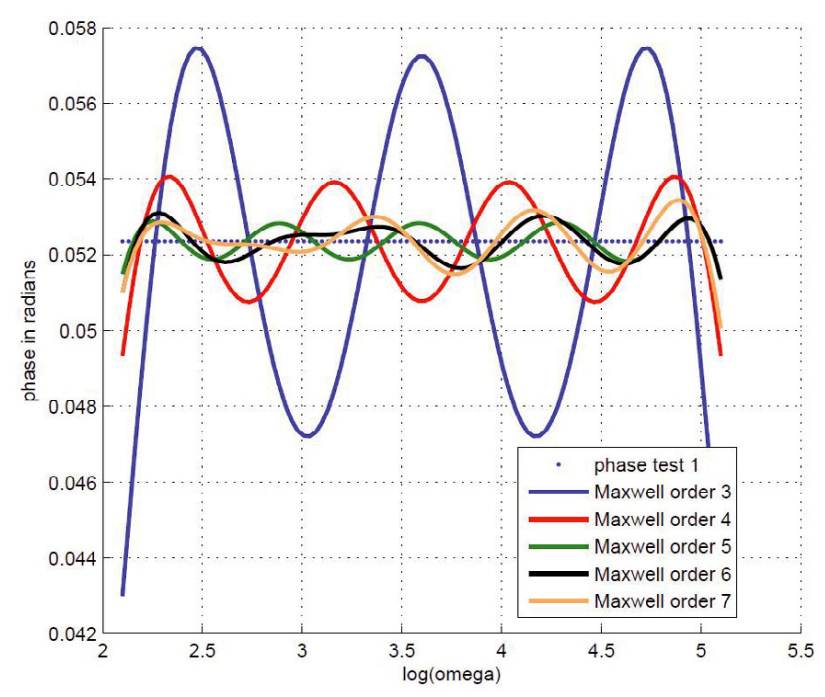

Fig. 16. Effet de l'ordre du modèle de Maxwell sur l'oscillation de la phase.

amélioré a été développé, entre temps, par Renaud mais n'a pas été utilisé pour ces travaux.

On observe le même effet de convergence que précédemment. Les écarts observés sont plus faibles que pour le nombre de modes de projection. D'autres essais ont montré que la valeur de la phase a un impact sur les écarts en fréquence mais peu sur les amortissements. Il est plus difficile de conclure sur le nombre de cellules à utiliser. En effet, l'augmentation de l'ordre impacte les écarts de manière positive et la taille du modèle et son conditionnement de manière négative. Un bon compromis est l'utilisation d'une cellule par décade plus une ou deux.

\subsection{Frein}

On utilise le même modèle de frein que dans la Section 4.2 . 


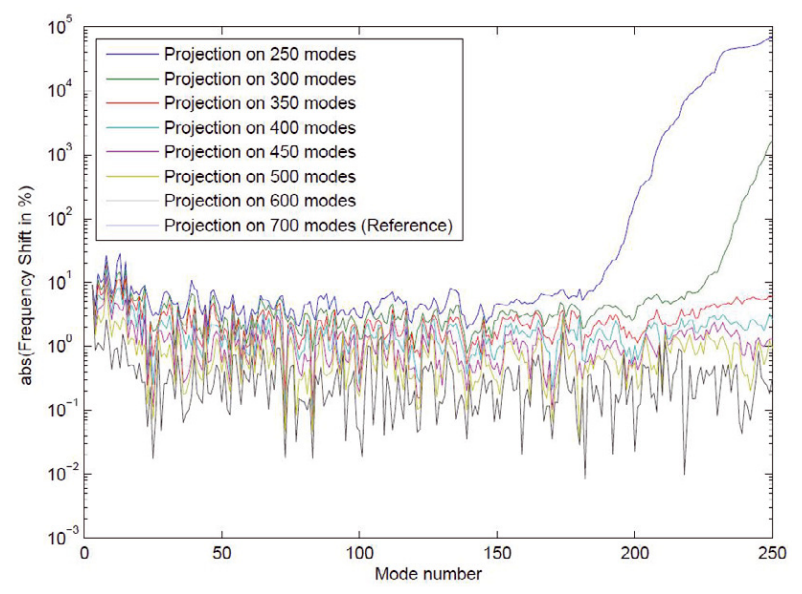

Fig. 17. Fréquences calculées par analyse modale complexe pour chaque modèle réduit.

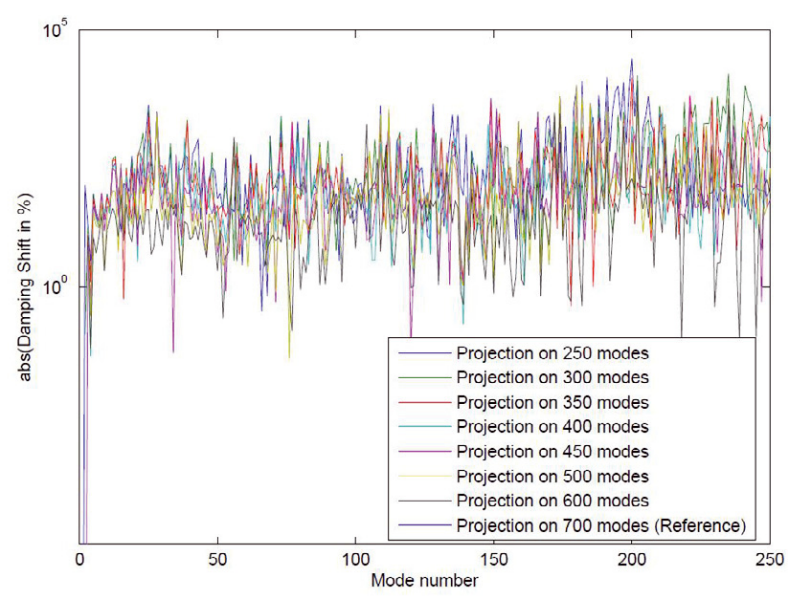

Fig. 18. Amortissements calculés par analyse modale complexe pour chaque modèle réduit.

\subsubsection{Influence du nombre de modes de projection}

La méthode est très similaire à celle de la plaquette. On extrait successivement 250, 300, 350, 400, 450, 500, 600 puis 700 modes réels et on projette le modèle sur ces bases. On calcule alors les modes complexes pour chaque modèle réduit. On prend en compte la viscoélasticité par un modèle de Maxwell généralisé d'ordre 5 dont les paramètres sont : MG[1 20000 [3 3 7] 5]. On obtient alors les fréquences et amortissements de tous les modes pour chaque modèle réduit.

On s'intéresse uniquement aux 250 premiers modes du frein. On trace l'écart en pour cent entre les résultats de chaque modèle réduit et les résultats du dernier (projeté sur 700 modes). Les résultats en fréquence sont illustrés sur la figure 17. Les résultats en amortissement sont illustrés par la figure 18. On utilise une vue avec une échelle logarithmique sur l'axe des ordonnées.

Comme dans le cas de la plaquette, on observe un effet de convergence vers la référence avec l'augmentation du nombre de modes de projection. Cet effet est clairement

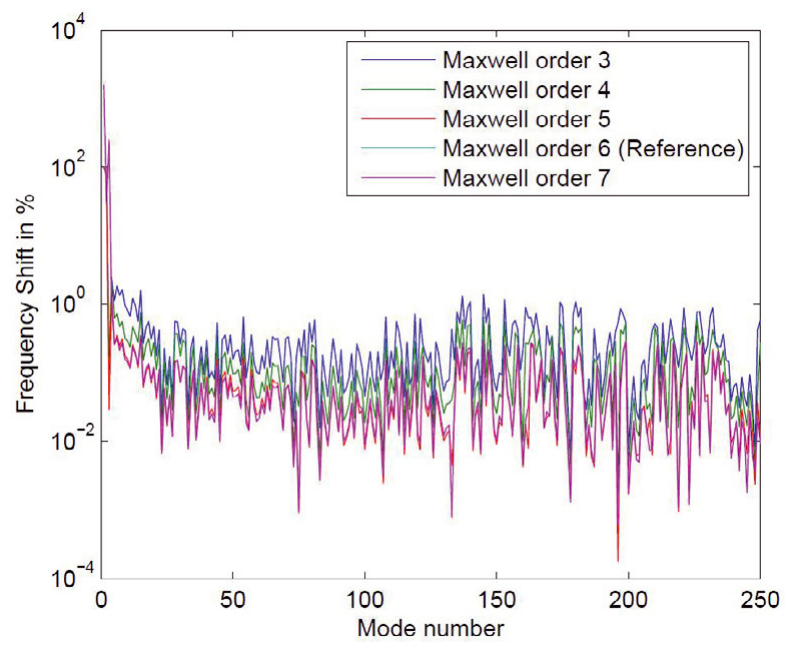

Fig. 19. Effet de l'ordre du modèle de Maxwell sur les fréquences.

visible pour les fréquences mais pas pour les amortissements pour lesquels l'écart mesuré est très élevé pour un grand nombre de modes et ce, quel que soit le nombre de modes de projection.

On peut considérer qu'une projection sur 400 modes réels est nécessaire pour étudier les 250 premiers modes complexes.

On peut également noter que les écarts peuvent être très importants (plus de $1000 \%$ ) dans le cas d'une troncature trop forte de la base de Ritz. Il faut donc rester très vigilant sur le nombre de modes observables pour une troncature donnée.

\subsubsection{Influence du nombre de cellules de Maxwell}

Afin de s'affranchir des problèmes liés à la réduction du modèle, on travaille avec un modèle projeté sur 500 modes réels. La méthode consiste alors à fixer un intervalle de fréquence de 3 décades (de $20 \mathrm{~Hz}$ à $20000 \mathrm{~Hz}$ ) et une phase constante à 3 degrés pour les matériaux de friction et 7 degrés pour le shim. On calcule successivement les fréquences et amortissements par analyse modale complexe en faisant varier l'ordre du modèle de Maxwell généralisé de 3 à 7 . On s'intéresse à l'influence de ce paramètre sur l'oscillation de la phase du modèle de Maxwell ainsi que son impact sur les fréquences et amortissements.

Regardons maintenant l'impact sur les fréquences et les amortissements de cette augmentation de l'ordre du modèle. Les figures 19 et 20 montrent les résultats obtenus en échelle logarithmique. Pour les mêmes raisons que pour la plaquette, l'ordre de référence n'est pas l'ordre le plus élevé.

De même que pour l'analyse sur le nombre de modes de projection, on obtient des écarts sur les amortissements qui atteignent des valeurs élevées, principalement pour les modes haute fréquence par rapport au domaine d'intérêt. La section suivante a pour but d'analyser ce phénomène. 


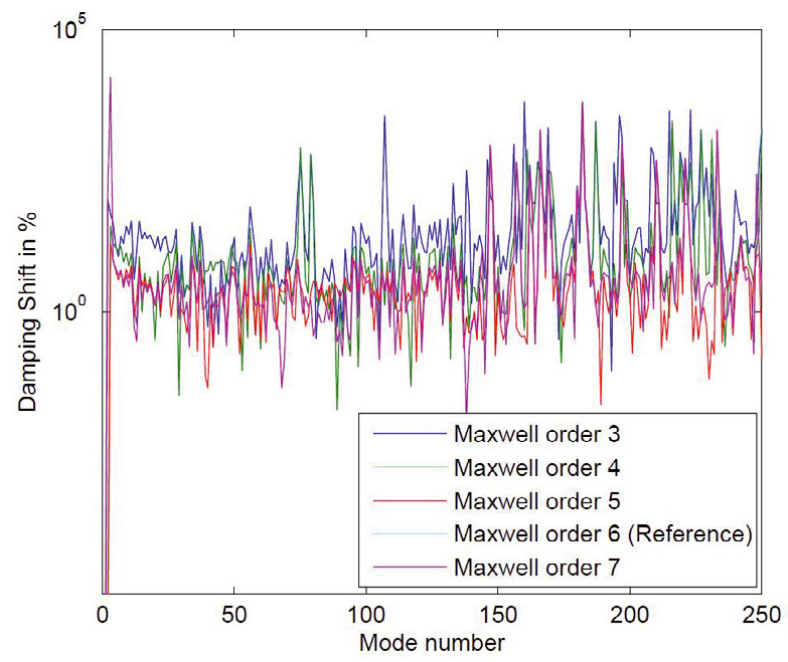

Fig. 20. Effet de l'ordre du modèle de Maxwell sur les amortissements.

Tableau 5. Fréquence et amortissement du mode 113 pour chaque ordre de Maxwell.

\begin{tabular}{ccc}
\hline Ordre Maxwell & Fréquence $(\mathrm{Hz})$ & Amortissement \\
\hline Ordre 3 & 8565,8 & $0,0103 \%$ \\
Ordre 4 & 8592,3 & $0,0078 \%$ \\
Ordre 5 & 8600,8 & $0,0030 \%$ \\
Ordre 6 & 8599,6 & $0,0030 \%$ \\
Ordre 7 & 8600,7 & $0,0030 \%$ \\
\hline
\end{tabular}

Tableau 6. Fréquence et amortissement du mode 121 pour chaque ordre de Maxwell.

\begin{tabular}{ccc}
\hline Ordre Maxwell & Fréquence $(\mathrm{Hz})$ & Amortissement \\
\hline Ordre 3 & 9127,5 & $0,0121 \%$ \\
Ordre 4 & 9171,9 & $0,0110 \%$ \\
Ordre 5 & 9183,8 & $0,0021 \%$ \\
Ordre 6 & 9184,5 & $0,0021 \%$ \\
Ordre 7 & 9183,6 & $0,0021 \%$ \\
\hline
\end{tabular}

\subsection{3 Étude des modes présentant un écart important sur l'amortissement}

On va restreindre l'étude au cas de l'analyse de l'effet du nombre de cellules de Maxwell. On va également limiter le nombre de modes étudiés à 2. On choisit les modes 113 et 121 .

Les résultats obtenus pour les deux modes retenus sont présentés dans les tableaux 5 et 6 .

Pour les deux modes 113 et 121, on constate qu'il y a un écart important sur l'amortissement des ordres 3 et 4 par rapport à l'ordre de référence (ordre 6). La figure 21 illustre ces observations.

L'écart observé vient d'une inversion de modes. Le mode 113 calculé pour un ordre 3 ou 4 du modèle de Maxwell n'est plus le même que le mode 113 calculé avec un modèle d'ordre 6 , de même que pour le mode 121 .

Pour confirmer cette interprétation, on trace les diagrammes de MAC.

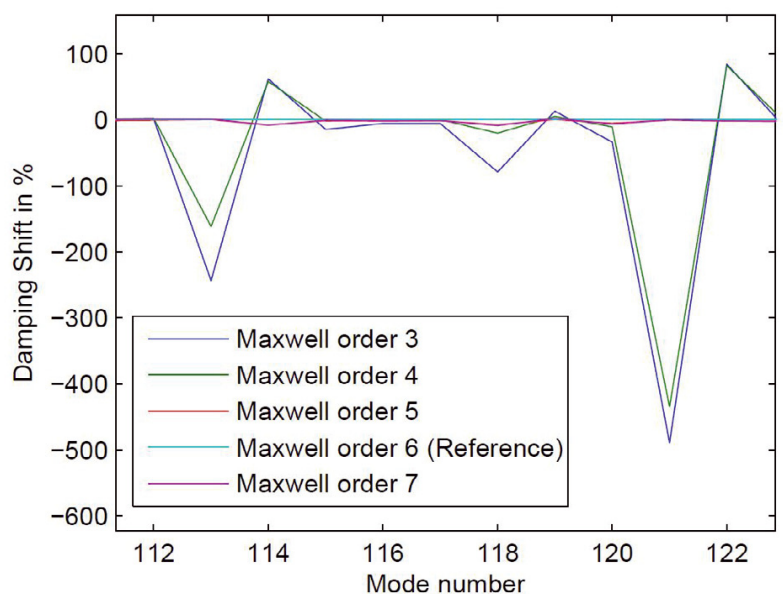

Fig. 21. Ecart en amortissement pour les modes 113 et 121 en fonction du nombre de cellules de Maxwell.

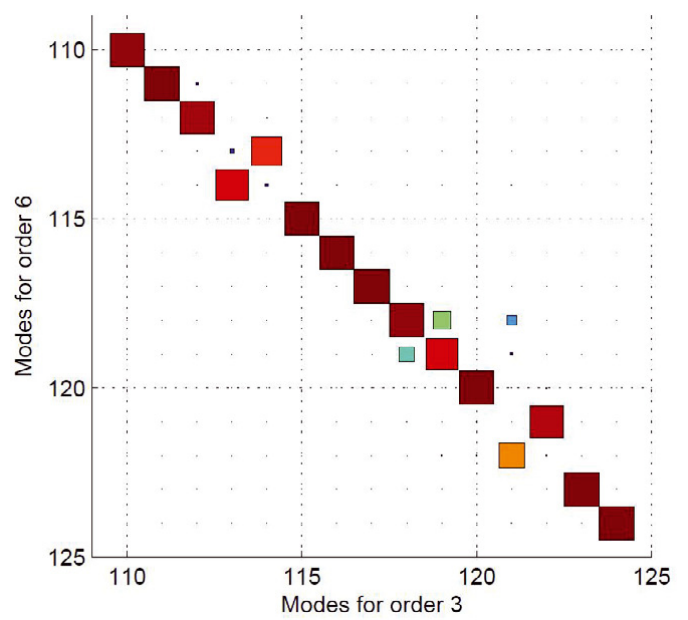

Fig. 22. Corrélation des modes ordre 3-ordre 6 .

- On trace la corrélation entre les modes calculés avec un modèle viscoélastique d'ordre 3 et ceux calculés avec un modèle viscoélastique d'ordre 6 (Fig. 22).

- On trace la corrélation entre les modes calculés avec un modèle viscoélastique d'ordre 4 et ceux calculés avec un modèle viscoélastique d'ordre 6 (Fig. 23).

On voit qu'il y a bien un problème dans l'ordre des modes. Après les avoir réordonnés correctement, on peut retracer la courbe d'écart en amortissement (Fig. 24). Les écarts observés sont désormais parfaitement cohérents, aussi bien pour le mode 113 que 121. Il suffit alors d'appliquer la même méthode à tous les modes présentant un écart trop important.

\section{Conclusion}

Au terme de cette étude, plusieurs objectifs ont été atteints :

- on dispose d'une modélisation mathématique du comportement viscoélastique d'un matériau. Ce modèle 


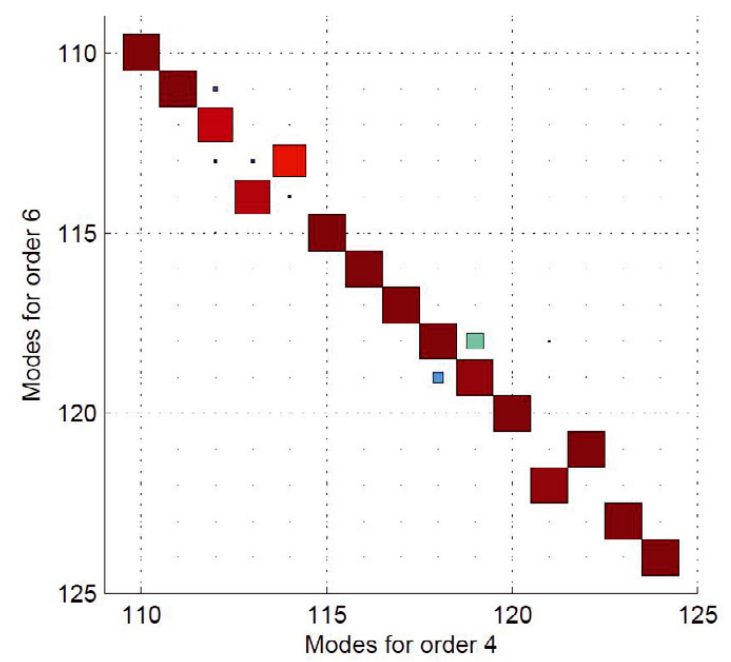

Fig. 23. Corrélation des modes ordre 4 - ordre 6 .

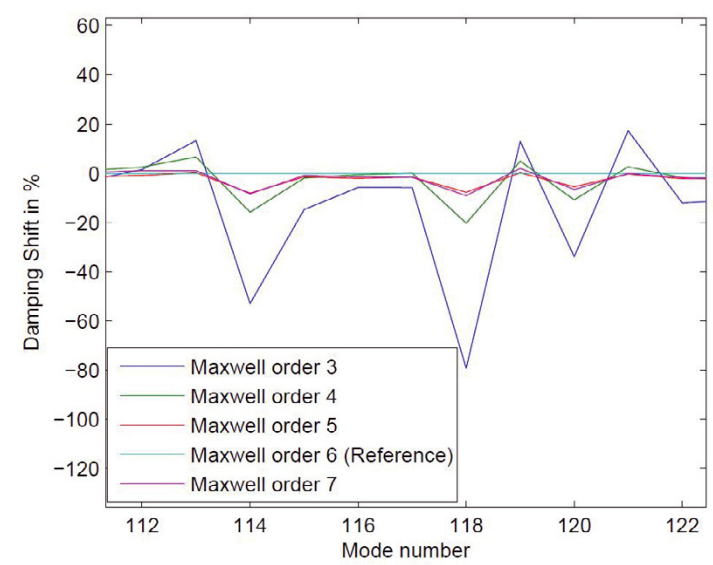

Fig. 24. Ecart en amortissement pour les modes 113 et 121 après correction de l'ordre des modes.

peut traduire un effet de rigidification en fonction de la fréquence ainsi que l'amortissement;

- on dispose d'une implémentation de ce modèle dans le cadre d'analyse modale complexe. Il est possible de traiter des problèmes avec des structures faisant intervenir plusieurs matériaux viscoélastiques à priori différents à condition qu'ils soient tous modélisés par un modèle de Maxwell généralisé;

- on dispose d'une passerelle logicielle développée en Python permettant la simulation mixte Abaqus ${ }^{\circledR} /$ Matlab ${ }^{\circledR}$ afin d'appliquer les modèles viscoélastiques aux modèles de frein Bosch;
- on a également mis en évidence l'impact important de la troncature modale et de l'ordre des modèles de Maxwell généralisé sur les résultats d'analyse modale complexe.

Les perspectives de cette étude sont l'utilisation de la méthode pour la prédiction des instabilités de freinage ainsi que pour l'aide au choix des matériaux. La prise en compte des effets de la température pourrait être une voie d'amélioration des méthodes de simulation utilisées par Bosch système de freinage.

\section{Références}

[1] N. Hoffmann, M. Fischer, R. Allgaier, L. Gaul, A minimal model for studying properties of the mode-coupling type instability in friction induced oscillations, Mechanics Research Communications 29 (2002) 197-205

[2] N.M. Kinkaid, O.M. O'Reilly, P. Papadopoulos, Automotive disc brake squeal, J. Sound Vib. 267 (2003) 105-166

[3] G. Fritz, J.-J. Sinou, J.-M. Duffal, L. Jézéquel, Investigation of the relationship between damping and mode-coupling patterns in case of brake squeal, J. Sound Vib. 307 (2007) 591-609

[4] G.A. Lesieutre, K. Govindswamy, Finite element modeling of frequency dependent and temperature-dependent dynamic behavior of viscoelastic materials in simple shear, Int. J. Solids Struct. 33 (1996) 419-432

[5] G.A. Lesieutre, Finite elements for dynamic modeling of uniaxial rods with frequency-dependent material properties, Int. J. Solids Struct. 29 (1992) 1567-1579

[6] G. Chevallier, L. Levy, J.-L. Dion, Étude des dispositifs de contrôle passif du crissement de frein, in Actes du colloque vibrations, chocs et bruits, Lyon, 2006

[7] A. Oustaloup, La commande CRONE. HERMES, 1991

[8] J.-L. Dion, Modélisation dynamique des suspensions hydroélastiques, Thèse de doctorat, Ecole Centrale de Paris, 1995

[9] E. Balmès, J.-M. Leclère, Viscoelastic vibration toolbox. User's guide, SDTools, 2007

[10] F. Renaud, J.-L. Dion, G. Chevallier, Identification des paramètres du modèle de Maxwell généralisé sur des courbes expérimentales de module et de phase, In Actes du colloque vibrations, chocs et bruits, Lyon, 2008

[11] ABAQUS/Std. Analysis User's Manual. Chap. 17.7.2. Abaqus v6.7 Documentation collection, 2007

[12] G. Fritz, J.-J. Sinou, J.-M. Duffal, L. Jézéquel, Effects of damping on brake squeal coalescence patterns - application on a finite element model, Mechanics Research Communications 34 (2007) 181-190 